\title{
Foreword
}

\section{Care of the Obstetric Patient: Mitigating Risk}

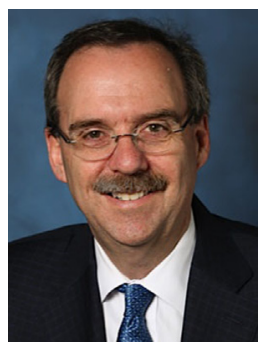

Lee $A$. Fleisher, MD Consulting Editor

Maternal morbidity and mortality have been increasing over the last several decades, and anesthesiologists need to be engaged in working with their obstetric colleagues in mitigating risk. In addition, there are wide disparities in complications when stratified by race and ethnicity. Most of us trained in obstetric anesthesia care during our residency and may not be aware of these trends or our ability to intervene. In this issue of Anesthesiology Clinics, the editors have assembled a series of articles that highlight the anesthesiologist's role in addressing peripartum complications and high-risk individuals. They have also included articles on ERAS (enhanced recovery after surgery) and ethics. It will allow us to assume shared accountability for peripartum care.

In order to commission an issue on obstetric anesthesia care, I have turned to colleagues at Massachusetts General Hospital (MGH). May Pian-Smith is Associate Professor of Anesthesia at Harvard Medical School and Division Chief for Quality and Safety in the Department of Anesthesia, Critical Care, and Pain Medicine at $\mathrm{MGH}$. Her research has been focused on improving the health care of women. She has pursued fellowship training in Medical Education and Patient Safety and lectures on fostering trust. Rebecca Minehart is Assistant Professor of Anaesthesia at Harvard Medical School and Program Director for Obstetric Anesthesia Fellowship at MGH. She has received grant funding for research in education from the Foundation for Anesthesia Education and Research as well as the Executive Committee on Teaching and Education at MGH. In addition, she is a member of the ASA's Interactive Computer-Based Education Editorial Board, and the Chair of the Society 
for Obstetric Anesthesia and Perinatology Fellowship Program Directors' Committee. Together, they have edited an important issue.

Lee A. Fleisher, MD Perelman School of Medicine at University of Pennsylvania 3400 Spruce Street, Dulles 680

Philadelphia, PA 19104, USA

E-mail address: 